\title{
Mecanismos de Ação Dos Bifosfonatos e sua Influência no Prognóstico do Tratamento Endodôntico
}

\section{Bisphosphonates Mechanisms of Action and its Influence in the Endodontic Treatment Prognosis}

\section{Felipe Cavalcanti Sampaio ${ }^{1}$, Heloísa Helena Pinho Veloso ${ }^{2}$, Danielle do Nascimento Barbosa ${ }^{3}$}

\begin{abstract}
The bisphosphonates are medicines widely used to treat bone mineral loss related diseases due to increasing of bone resorption, being the first choice drugs for osteoporosis treatment and other chronic bone diseases, as Paget disease or osteolytic bone metastasis. These medicines act, direct or indirectly, on osteoclasts and osteoblasts, which results in bone remodeling decreasing, besides presenting inhibitor effects on the inflammatory modulators, what may influence the bone wounds repair process. Within this context, it is proposed to present, by means of a literature review, bisphosphonates mechanisms of action and its influence on the endodontic treatment prognosis, especially with regard to the apical periodontitis repair process. It was concluded bisphosphonates show potential, from their mechanisms of action, potential to influence in the apical periodontitis repair process, and more studies are required to establish a cause-effect relation between bisphosphonates use and the apical periodontitis repair process.
\end{abstract}

Keywords: Diphosphonates; Bone remodeling; Periapical Periodontitis.

\section{Resumo}

Os bifosfonatos são medicamentos amplamente utilizados no tratamento de doenças relacionadas à perda de mineral ósseo devido ao aumento da reabsorção óssea, sendo as drogas de primeira escolha no tratamento para osteoporose e demais doenças ósseas crônicas, como doença de Paget ou metástases ósseas osteolíticas. Estes medicamentos atuam, direta ou indiretamente, sobre os osteoclastos e osteoblastos, o que resulta na diminuição da remodelação óssea, além de apresentarem efeitos inibitórios sobre mediadores da inflamação inflamatório, podendo influenciar o processo de reparo de lesões ósseas. Dentro desse contexto, propôs-se a apresentar, por meio de uma revista da literatura, os mecanismos de ação dos bifosfonatos e sua influência no prognóstico do tratamento endodôntico, principalmente no que concerne ao reparo de periodontites apicais. Concluiu-se que os bifosfonatos demonstram, a partir de seus mecanismos de ação, potencial para influenciar no processo de reparo de periodontites apicais, e que mais estudos são necessários a fim de estabelecer uma relação causa-efeito entre o uso de bifosfonatos e o processo de reparo de lesões ósseas.

Palavras-chave: Difosfonatos; Remodelação óssea; Periodontite Periapical.
${ }^{1}$ Especialista em Endodontia, Pós-graduando nível Mestrado pela Universidade Federal de Goiás - UFG

2 Doutora em Endodontia, Professora Adjunta do Departamento de Odontologia Restauradora da Universidade Federal da Paraíba - UFPB

${ }^{3}$ Mestre em clínicas odontológicas. Universidade Estadual de Paraíba - UEPB

Correspondência: Felipe Cavalcanti Sampaio

Endereço: Décima Primeira Avenida, 334, Apto.208 - CEP 74605-060, Goiânia GO, Brasil

Fone: (62) 9646-7334

E-mail: felipecavalcantisampaio@yahoo.com

Data de Submissão: 06/02/2011

Data de Aceite: 25/07/2011

\section{Introdução}

A redução da concentração do cálcio pode acarretar vários agravantes, como afetar a transmissão de impulsos nervosos. Para impedir tais efeitos, o organismo possui mecanismos de alta sensibilidade que regulam a concentração de cálcio. O principal deste é a reabsorção óssea, que aumentará a quantidade de íons cálcio no líquido extracelular e mantendo assim as funções normais do organismo. Entretanto, com a manutenção da condição patológica crônica, o osso perderá densidade mineral e, portanto, tornar-se-á poroso e susceptível a fraturas, denominado osteoporose (GUYTON; HALL, 2006).

A osteoporose é atualmente um problema de saúde pública mundial. No Brasil, o Sistema Único de Saúde reportou de Janeiro a Outubro de 200920.778 internações em decorrência de fraturas em pacientes com osteoporose, gastando-se 57,61 milhões de reais (BRASIL, 2010).

Além da osteoporose, outras patologias estão associadas à elevada reabsorção óssea, como: a terapia com glicocorticoides pode causar uma osteoporose secundária (ANGELI et al., 2006; CANALIS et al., 2007; WEINSTEIN 2008; WEINSTEIN, 2011); a doença de Paget do osso, uma desordem de etiologia

desconhecida que envolve alterações na remodelação óssea, levando a complicações como dor óssea, deformidades esqueléticas e fraturas (CUNDY; BOLLAND, 2008; SILVERMAN, 2008); metástases osteolíticas, comuns em neoplasias de mama, próstata ou pulmão (CHEN et al., 2006; RENTSCH et al., 2009; GUISE et al. 2010; LIPTON, 2010; GROSSMAN et al., 2011); a hipercalcemia, uma complicação metabólica comum de patologias malignas (BURT; BRENNAN, 1980; VASSILOPOULOU-SELLIN et al., 1993; MAKRAS; PAPAPOULOS, 2009 SARGENT; SMITH, 2010).

Os bifosfonatos, análogos do pirofosfato quimicamente e em sua afinidade com a hidroxiapatita do osso, são drogas utilizadas amplamente no tratamento de doenças que afetam o metabolismo ósseo associadas à excessiva reabsorção (RODAN; FLEISCH, 1996; RODAN, 1998; KATZ, 2005; FERREIRA JÚNIOR et al., 2007; BRANDÃO et al., 2008; CARTSOS et al., 2008; CARVALHO et al. 2008; SHINODA et al., 2008; DORE et al., 2009; TOUSSAINT et al., 2009). Foram primeiramente sintetizados em 1865. Inicialmente, foram utilizados em procedimentos industriais, como agentes anticorrosivos e anti-incrustantes. Após a descoberta de que tinham influência no controle da formação e dissolução do fosfato de cálcio in vitro, bem como na mineralização e reabsorção 
óssea in vivo, foram desenvolvidos e utilizados no tratamento de doenças ósseas (RODAN; FLEISCH, 1996).

Como são drogas amplamente utilizadas na atualidade, o cirurgião-dentista deve estar atento para os efeitos que esta droga venha a causar no paciente, podendo afetar os procedimentos e o prognóstico do tratamento odontológico.

Durante o processo de reparo de uma periodontite apical existe uma sequência de eventos que pode ser originada por agentes infecciosos e/ou por agentes externos não infecciosos, como traumatismos ou procedimentos iatrogênicos. O processo de reparo se constitui basicamente de quatro fases: injúria, inflamação, proliferação e remodelação (ESTRELA, 2009).

Por interferirem na remodelação óssea, assim como agirem inibindo os mediadores da inflamação, os bifosfonatos podem influenciar o processo de reparo de lesões ósseas. Deste modo, poderiam influenciar $o$ prognóstico do tratamento endodôntico, no caso das periodontites apicais, nas quais há presença de processos infecciosos crônicos com formação de lesão inflamatória (ALLEN et al., 2001; HIKITA et al., 2009; TOUSSAINT et al., 2009; AGUIRRE et al., 2010; KOBAYASHI et al., 2010).

Nesse sentido, o objetivo do presente estudo é apresentar através de uma revisão de literatura os mecanismos de ação dos bifosfonatos e sua influência no prognóstico do tratamento endodôntico, principalmente no que concerne ao reparo de periodontites apicais.

\section{Métodos}

Foi realizado um levantamento bibliográfico sobre os mecanismos de ação dos bifosfonatos e sua influência no prognóstico das periodontites apicais após o tratamento endodôntico. Esta pesquisa contemplou diversas publicações científicas publicados em âmbito nacional e internacional. A seleção do conteúdo foi baseada em conformidade com a limitação dos assuntos aos objetivos do trabalho. Os meios utilizados para o levantamento da literatura foram os canais de busca: Pubmed, BBO, Scielo e Lilacs, que possibilitam acesso tanto aos artigos mais recentes como também aos trabalhos clássicos referentes aos temas de interesse. Os trabalhos foram buscados no intervalo de data de publicação de 2001 a 2011. Por se tratar de revisão crítica da literatura, foram incluídos no trabalho apenas textos de pesquisa ou revisão que abordassem o tema e fornecessem informações acerca dos mecanismos de ação dos bifosfonatos e/ou sua utilização e efeitos nos tratamentos para os quais são recomendados, assim como aqueles que relacionassem este tipo de medicamento à endodontia.

\section{Revista da Literatura}

Bifosfonatos são análogos sintéticos do pirofosfato nos quais a ponte de oxigênio é substituída por um carbono ( $P-C-P)$, formando duas cadeias principais $\left(R_{1}\right.$ e $\left.R_{2}\right)$. A cadeia longa $R_{2}$ determina a potência antirreabsortiva e o mecanismo de ação farmacológico. Assemelham-se ao pirofosfato ainda em sua ligação com a hidroxiapatita do osso. (RODAN; FLEISCH, 1996; RODAN, 1998; FERREIRA JÚNIOR et al., 2007; BRANDÃO et al., 2008; CARTSOS et al., 2008; CARVALHO et al. 2008; SHINODA et al., 2008; DORE et al., 2009; TOUSSAINT et al., 2009).

A presença de nitrogênio na cadeia $R_{2}$ faz com que estas drogas sejam classificadas em bifosfonatos nitrogenados ou aminobifosfonatos, representados por pamidronato, alendronato, ibandronato, risedronato e zoledronato, e bifosfonatos não nitrogenados, que são etidronato, tiludronato e clodronato. São conhecidos por terem meia vida longa, podendo durar meses a anos, por se aderirem ao mineral ósseo e lá permanecerem até que o sítio ósseo em que se encontra seja reabsorvido (FERREIRA JUNIOR et al. 2007; RODAN, 1998).
Estes compostos são utilizados no tratamento de doenças que afetam o metabolismo ósseo associadas à excessiva reabsorção, como osteoporose pós-menopausa ou causada pelo tratamento com glicocorticoides, doença de Paget, e na oncologia, como nos casos de hipercalcemia maligna ou metástases ósseas osteolíticas, estas últimas comuns em câncer de mama, pulmão ou próstata (RODAN; FLEISCH, 1996; MELO; OBEID, 2005; FERREIRA JÚNIOR et al., 2007; BRANDÃO et al., 2008; CARTSOS et al., 2008; SHINODA et al., 2008; SILVERMAN, 2008; DORE et al., 2009; TOUSSAINT et al., 2009; NEVILLE-WEBBE; COLEMAN, 2010; ABUGHAZALEH; KAWAR, 2011; TERPOS et al., 2011).

O uso de bifosfonatos pode estar associado a reações adversas, a maioria focada no sistema digestório, além de dores ósseas, musculares, articulares e reações alérgicas (FERREIRA JUNIOR et al., 2007; LEVIN et al., 2007). Recentemente, foi identificada uma nova complicação bucal, de interesse para a classe odontológica denominada osteonecrose dos maxilares ou osteonecrose associada aos bifosfonatos. Trata-se de uma séria reação adversa que acomete, por mecanismo ainda desconhecido, os ossos maxilares, provocando grande destruição tecidual (FERREIRA JÚNIOR et al., 2007; KHOSLA et al., 2007; LEVIN et al., 2007; COLELLA et al., 2009; ABUGHAZALEH; KAWAR, 2011).

Desde 1995 os bifosfonatos têm assumido um papel predominante no tratamento da osteoporose e das alterações do metabolismo ósseo associadas a neoplasias. Com efeito, desde a sua introdução no mercado, têm sido fármacos de eleição na terapêutica de patologias como o mieloma múltiplo e prevenção e terapêutica de metástases ósseas (CARVALHO et al., 2008; ABUGHAZALEH; KAWAR, 2011).

Apenas em 2004 o laboratório responsável pela introdução no mercado dos bifosfonatos pamidronato e zoledronato alertou os profissionais de saúde para os riscos associados ao desenvolvimento de osteonecrose dos maxilares, fato que em 2005 incluiu a todos os bifosfonatos, inclusive as formas orais, como potenciais desencadeadores dos processos de osteonecrose (CARVALHO et al., 2008; ABUGHAZALEH; KAWAR, 2011).

\section{Mecanismos de Ação dos Bifosfonatos}

A carga e o volume dos bifosfonatos limita sua penetração na membrana celular, o que pode explicar a baixa absorção no intestino (de 1\% a $7 \%$ ). Uma vez absorvidos, têm elevada afinidade pelo mineral ósseo, ligando-se ao cálcio nos cristais de hidroxiapatita, com uma meia-vida variável no esqueleto que pode chegar até 10 anos. O restante absorvido não vinculado ao osso é rapidamente excretado inalterado (RODAN; FLEISCH, 1996; TOUSSAINT et al., 2009).

Tal afinidade pela hidroxiapatita explica a específica ação farmacológica desses compostos sobre os tecidos minerais. Com efeito, são depositados onde o mineral ósseo é exposto aos líquidos circundantes, especialmente nos sítios de remodelação óssea. Nos tecidos, a ação de todos os bifosfonatos ativos parece ser semelhante: redução da remodelação óssea (RODAN; FLEISCH, 1996).

Além da reabsorção, a formação óssea também é reduzida, como evidenciado por uma redução na superfície de neoformação. Como não há evidência de atividade osteoblástica reduzida em sítios de formação óssea individuais, conclui-se que a redução da superfície total de formação óssea é secundária à diminuição da reabsorção (RODAN; FLEISCH, 1996; RODAN, 1998).

Uma explicação para o ganho de mineral ósseo é que a redução na reabsorção óssea não é imediatamente seguida pela diminuição na formação óssea, já que a formação em sítios de remodelação iniciadas antes do tratamento irá proceder até sua conclusão. Isso irá produzir um ganho relativamente rápido (de 4 a 6 meses) da massa óssea. Outra explicação é que, após a diminuição do volume de remodelação, o osso recém-formado terá menos chance de ser neoformado e terá, portanto, mais tempo 
para completar sua mineralização. Foi demonstrado que $70 \%$ da mineralização do novo osso formado ocorre dentro das primeiras semanas, mas o enriquecimento mineral continua por muito tempo. Portanto, ossos "mais velhos", com maior tempo entre suas remodelações, têm um maior teor de minerais (RODAN; FLEISCH, 1996; RODAN, 1998; RUSSELL et al., 2008; ALLEN et al., 2010; ALLEN, 2011).

Ao nível celular há concordância geral que o alvo final da ação dos bifosfonatos é o osteoclasto, direta ou indiretamente, esta última através das células moduladoras (RODAN; FLEISCH, 1996; RODAN, 1998). In vitro, vários bifosfonatos inibem a diferenciação dos osteoclastos oriundos da medula óssea (HUGHES et al., 1989). Em um sistema de co-cultura de células derivadas de osteoblastos de ratos e células da medula óssea, o alendronato inibiu osteoclastogênese induzida por vitamina $D_{3}$. São totalmente eficazes neste sistema quando adicionado imediatamente antes da fusão dos precursores mononucleares dos osteoclastos para formar células multinucleadas, também verificado in vivo (SCHMIDT et al., 1996). No entanto, vários fatos sugerem que este não é o seu único modo de ação in vivo, uma vez que, após a administração de bifosfonatos, o número de osteoclastos multinucleados na superfície óssea normalmente aumenta primeiro, mas as células parecem inativas. Só mais tarde, após a administração crônica, é que o número de osteoclastos diminui (BOONEKAMP et al., 1986; RODAN; FLEISCH, 1996; RODAN, 1998).

Os efeitos sobre o recrutamento de osteoclastos podem ser diretos e/ou indiretos. É normalmente aceito que as células da linhagem osteoblástica podem controlar o recrutamento e atividade dos osteoclastos nos termos fisiológicos e condições patológicas. Foi demonstrado que quando as células osteoblásticas de rato são breve ou continuamente expostas a baixas concentrações de bifosfonatos, o seu meio apresenta ao menos um fator que reduz a reabsorção óssea osteoclástica. Os osteoblastos em co-cultura com células da medula óssea seguido de tratamento com risedronato mostraram marcação de supressão de diferenciação celular. Desde que os osteoblastos expressam RANKL, essencial para a diferenciação dos osteoclastos, os efeitos do risedronato nesta diferenciação pode estar relacionada à diminuição da expressão de RANKL ou expressão aumentada de osteoprotegerina (OPG), inibidor da diferenciação de osteoclastos (RODAN; FLEISCH, 1996; RODAN, 1998; KWAK et al., 2009).

Weinstein et al. (2009) verificaram a formação de células gigantes e sua relação ao uso dos aminobifosfonatos. Segundo o estudo, espera-se que haja redução não só de osteoclastos, como também do osteóide, de osteoblastos e da neoformação óssea na terapia com alendronato.

Além da formação de osteoclastos, há também inibição de atividade destes na superfície óssea, fortemente apoiada como parte do mecanismo de ação dos bifosfonatos pelo fato de que osteoclastos mostram mudanças na sua morfologia tanto in vitro como in vivo (SCHENCK et al., 1973; SATO; GRASSER, 1990; MURAKAMI et al., 1995). Estas incluem alterações no citoesqueleto, especialmente a actina, e na borda vilosa ou pregueada, membrana característica dos osteoclastos ativos (PLASMANS et al. 1980; SATO et al. 1991)

A liberação dos bifosfonatos ocorre principalmente quando o osso onde estão é reabsorvido, respondendo por sua longa meia-vida. Pode então ser fagocitado pelos osteoclastos e, em seguida, retornar à circulação, onde pode ser submetido à excreção renal ou permanecer na circulação até ser reincorporado ao osso. Esta "reciclagem" dos bifosfonatos pode explicar porque, após a interrupção do tratamento, pequenas quantidades de bifosfonatos pode ser detectada na urina até mesmo após 10 anos (RODAN; FLEISCH, 1996; RODAN, 1998; CARTSOS et al., 2008; KWAK et al., 2009; TOUSSAINT et al., 2009).

A supressão da ação dos osteoclastos pode ocorrer devido por meio da inibição da enzima farnesil pirofosfato sintase (FPP sintase), enzima da via do mevalonato cuja inibição impede a função celular normal e sobrevivência dos osteoclastos (KWAK et al., 2009; TOUSSAINT et al., 2009). Outros dois grupos de enzimas se mostraram inibidas por bifosfonatos: esqualeno sintase, e as proteína tirosina fosfatases (PTPs), o que afetaria a biossíntese do colesterol, cuja relação na formação da borda pregueada é incerta, e as vias de transdução iniciadas pelos fatores de crescimento, insulina e outros estímulos (RODAN; FLEISCH, 1996; RODAN, 1998; RESZKA; RODAN, 2003).

Além da atuação sobre os osteoclastos, os efeitos dos estimuladores de reabsorção óssea testados, que incluíram LPS, $\mathrm{PGE}_{2}, \mathrm{IL}-1 \beta$ e TNF- $\alpha$, foram inibidos por bifosfonatos. Estes achados sugerem que estas drogas inibem a reabsorção óssea por inibir a estimulação dos osteoclastos (SHINODA et al., 2008; KWAK et al., 2009).

\section{Osteonecrose dos Maxilares Associada aos Bifosfonatos}

A osteonecrose é definida como uma exposição de osso maxilar ou mandibular por meio de lesões gengivais que não regeneram (KHOSLA et al., 2007). Pode ter várias etiologias e ser multifatorial. Pode resultar de formas necrosantes de periodontopatias, infecções, patologias malignas e medicamento para seus tratamentos, radiação e outras patologias ósseas (ABUGHAZALEH; KAWAR, 2011).

A osteonecrose dos maxilares é uma séria complicação, de difícil tratamento, podendo levar a óbito, cujos sintomas incluem dor intensa e perda de grandes porções de mandíbula e/ou maxila (ABUGHAZALEH; KAWAR, 2011).

Os primeiros relatos de osteonecrose dos maxilares associada aos bifosfonatos, datados de 2003, envolveram doentes que faziam uso por via intravenosa para tratamento de doenças ósseas metastáticas. Alguns casos têm sido associados ao uso por via oral, muito utilizada para osteoporose e osteopenia (FERREIRA JUNIOR et al., 2007; CARVALHO et al., 2008).

Pacientes são considerados portadores de osteonecrose associada aos bifosfonatos quando apresentam três características fundamentais: terem sido submetidos a tratamento atual ou prévio com bifosfonatos; apresentarem osteonecrose na região maxilofacial, caracterizada por exposição de osso, por mais de oito semanas; e não terem sido submetidos à radioterapia nos maxilares (FERREIRA JUNIOR et al., 2007; CARVALHO et al., 2008; COLELLA et al., 2009; ABUGHAZALEH; KAWAR, 2011).

As lesões de osteonecrose dos maxilares surgem, geralmente, como ulcerações na mucosa com exposição óssea subjacente e com dor associada, embora em um terço dos casos seja indolor. Apresentam localização predominante na mandíbula $(65 \%)$, embora também existam relatos de casos na maxila $(26 \%)$, em ambos (9\%), e no palato. A localização preferencial parece estar relacionada com as características anatômicas e fisiológicas da mandíbula, designadamente a sua menor vascularização. Estas lesões tornam-se persistentes, não reagindo aos tratamentos convencionais (FERREIRA JUNIOR et al., 2007; CARVALHO et al., 2008; ABUGHAZALEH; KAWAR, 2011).

Nas fases iniciais não se detectam manifestações radiográficas e normalmente os pacientes não apresentam sintomas. Quando a exposição óssea torna-se mais extensa, o sinal clínico mais comum é a presença de rugosidades em tecido mole que rodeiam a área do osso necrótico, podendo haver indícios de infecção secundária. Em estágios mais avançados, pode haver queixa de dor intensa, com áreas de parestesia (FERREIRA JUNIOR et al., 2007; ABUGHAZALEH; KAWAR, 2011).

As causas da osteonecrose dos maxilares associada aos bifosfonatos ainda são obscuras, mas parecem advir de uma complexa interação entre o redução da remodelação óssea, trauma local, infecção, hipovascularização e o uso de bifosfonatos. Fatores sistêmicos como idade avançada, diabetes mellitus, baixa de hemoglobina, obesidade, imunossupressão, câncer, uso de outras medicações, como agentes quimioterápicos e corticosteroides, e diálise renal também parecem estar relacionados (FERREIRA JUNIOR et al., 2007; EDWARDS et al., 2008; ABUGHAZALEH; KAWAR, 2011) 
É cada vez mais aceito que a supressão da remodelação óssea tem papel significante na patofisiologia da osteonecrose dos maxilares. A evidência que sustenta esta hipótese vem de pacientes não tratados com bifosfonatos. O Denosumab, um inibidor da RANKL, suprime a remodelação a nível similar aos bifosfonatos, e recentes ensaios clínicos e relatos de casos tem mostrado associação do uso deste medicamento à osteonecrose dos maxilares (AGHALOO et al., 2010; ALLEN, 2011).

Os pacientes que fazem uso de bifosfonatos administrados por via parenteral são mais susceptíveis à osteonecrose dos maxilares do que os tratados por via oral, diretamente relacionado à dose e a potência da droga utilizada. Pacientes que são submetidos a cirurgias odontológicas concomitantes apresentam risco 7 vezes maior de adquirir a patologia. Infecções periodontais, periapicais e pericoronais são fatores predisponentes. Fumantes, etilistas e com higiene oral deficiente também têm risco aumentado de desenvolvê-la (FERREIRA-JUNIOR et al., 2007; EDWARDS et al., 2008; RIZZOLI et al., 2008; ABUGHAZALEH; KAWAR, 2011).

A associação entre procedimentos cirúrgicos odontológicos e a osteonecrose dos maxilares associada aos bifosfonatos pode estar relacionada à interferência do processo de reparo pós-cirúrgico. Um a das etapas para reparo de feridas ósseas é a remodelação, suprimida por estes medicamentos (ALLEN, 2011).

Marx et al. (2005) concluíram que a completa prevenção da osteonecrose dos maxilares no momento não é possível. No entanto, os cuidados odontológicos prévios ao tratamento reduzem a incidência desta patologia, e a escolha de procedimentos odontológicos não-cirúrgicos podem reduzir a ocorrência de novos casos.

Ripamonti et al. (2009) investigaram a ocorrência de osteonecrose dos maxilares antes e depois de medidas odontológicas preventivas em pacientes submetidos à terapia com bifosfonatos. As medidas preventivas foram a exodontia de dentes com mobilidade (nível 3 e 4), tratamento periodontal, realização de tratamento de higienização pelo profissional, tratamento restaurador ou endodôntico, quando necessários. Observou-se uma redução na incidência de $3,2 \%$ para $1,3 \%$ na comparação pré e pós medidas preventivas.

Apesar de seu difícil controle, existem algumas estratégias sugeridas de tratamento da osteonecrose dos maxilares. O uso de antissépticos bucais, antibioticoterapia sistêmica, além de procedimentos cirúrgicos, como curetagem e ressecção ósseas, têm sido realizados como estratégias de tratamento. As lesões também têm sido tratadas de outras formas, incluindo terapia de oxigenação hiperbárica e ozônio (MELO; OBEID, 2005; FERREIRA JÚNIOR et al., 2007; EDWARDS et al., 2008; FREIBERGER, 2009).

\section{Bifosfonatos na Endodontia e Reparo de Lesões}

Uma preocupação crescente sobre a osteonecrose dos maxilares associada aos bifosfonatos levou ao desenvolvimento de diretrizes para o tratamento dos pacientes que fazem uso destes medicamentos por organizações profissionais como a American Dental Association (ADA). Para os dentes severamente deteriorados, o tratamento endodôntico não-cirúrgico é preferível à exodontia quando os pacientes relatam uma história de uso de bifosfonato intravenoso ou uso prolongado de bifosfonato oral. Foi relatado que o tratamento endodôntico está relacionado à osteonecrose associada aos bifosfonatos em apenas $0,8 \%$ dos casos (MARX et al., 2005; HSIAO et al., 2009).

Trata-se de uma patologia com importantes implicações clínicas, pois os procedimentos cirúrgicos (incluindo exodontias ou tratamento endodôntico cirúrgico) são contraindicados, e nesses pacientes a dor pode imitar a de origem odontogênica, o que pode levar ao diagnóstico incorreto e consequente tratamento por uma causa odontogênica inexistente (KATZ, 2005).
Além da osteonecrose dos maxilares, o reparo de lesões ósseas pode ser influenciado por estas drogas. Estudos em animais começaram a investigar os efeitos dos bifosfonatos no reparo de lesões da cavidade bucal. Em roedores, o reparo inicial pós-exodontia (entre 5 e 10 dias) foi afetado, com os animais tratados com bifosfonatos tendo menos da metade do osso no alvéolo comparado com os controles (HIKITA et al., 2009; AGUIRRE et al., 2010; KOBAYASHI et al., 2010). Estes resultados estão associados à reduzida angiogênese (AGUIRRE et al., 2010; KOBAYASHI et al., 2010). Quando foram avaliados períodos mais longos (14 a 70 dias), as diferenças entre os roedores tratados com bifosfonatos e os controles não existiram, apresentando quantidade similar de osso e número de vasos sanguíneos no local, o que sugere que os efeitos são transitórios com relação à formação de osso e angiogênese (HIKITA et al., 2009; AGUIRRE et al., 2010).

Estudo com galinhas verificou a formação óssea induzida por bifosfonatos (pamidronato) no período de 7 dias. Verificaram que houve efeito positivo do uso de bifosfonatos na formação óssea (WEI et al., 2010).

Estudo em cães tratados com zoledronato encontrou resultados similares de formação óssea 4 semanas pós-exodontia quando comparados aos controles. Após 8 semanas a quantidade de osso formado também foi semelhante entre os grupos. Entretanto, o tipo de osso formado foi significativamente diferente. Nos animais do grupo controle, não tratados, a maior parte do osso trabecular no alvéolo foi remodelado e na região apical formou-se uma barreira cortical espessa e contínua. Já nos animais tratados com zoledronato, o osso trabecular permanecia no alvéolo e havia pouca formação de cortical na região apical (ALLEN et al. 2001).

Ainda não existe um modelo de estudo em animais que permita obter resultados confiáveis e comparáveis em relação aos efeitos do uso dos bifosfonatos no processo de reparo de lesões pós-cirúrgicas (ALLEN, 2011).

Avaliou-se a eficácia do etidronato como medicação intracanal nos canais radiculares de dentes de macaco avulsionados quanto à reabsorção radicular e a anquilose, comparando-o ao hidróxido de cálcio. Em geral, o uso de bifosfonatos resultou em um resultado inferior do que o cálcio hidróxido tanto em termos de reabsorção radicular como também de anquilose quando utilizado como medicação intracanal (THONG, 2009).

Komatsu et al. (2008) avaliaram a longo prazo efeitos inibitórios do alendronato tópico sobre a reabsorção em dentes reimplantados. Os efeitos inibitórios do alendronato tópico foram mantidos para a reabsorção óssea e radicular, mas não quanto à anquilose e mineralização da polpa, em dentes reimplantados por 4 meses. $O$ alendronato tópico também estimulou a formação óssea ao redor dos dentes reimplantados.

Estudo visando verificar o efeito do uso de bifosfonatos tópico na reabsorção induzida por movimentação ortodôntica observou que, em exames microradiográficos e histológicos, o uso experimental de anel elástico induziu reabsorção óssea vertical e horizontal severa, enquanto que a administração tópica de bifosfonatos nestes casos preveniu significativamente a perda óssea alveolar (SHINODA et al. 2008).

Xiong et al. (2007) realizaram estudo para avaliar se a deficiência de estrogênio e a terapia com alendronato sistêmico afeta a perda de osso alveolar em lesões periapicais. Imagens radiográficas revelaram que após a exposição pulpar para induzir periodontite apical, houve reabsorção do osso alveolar evidente nas áreas periapical. Ovariectomia resultou em um maior tamanho da lesão periapical, enquanto que após a administração do alendronato diário a perda óssea na região periapical foi claramente reduzida.

Xiong et al. (2010) investigaram os efeitos de alendronato administrado sistemicamente na reabsorção óssea alveolar e angiogênese em ratos submetidos à lesão periapical experimental, avaliados em dois períodos de tempo (2 e 4 semanas). Encontraram inibição de reabsorção óssea nos dois períodos analisados, sem levar a decréscimo na angiogênese. 
Hsiao et al. (2009) avaliaram o reparo de periodontites apicais em pacientes em uso de bifosfonatos por via oral após a terapia de canal radicular. No grupo com bifosfonatos, $26,5 \%$ das lesões foram consideradas não apresentando sinais de reparo, enquanto os casos controle não havia sinais de reparo em $18,4 \%$ dos casos. Não houve diferença estatisticamente significativa entre os grupos. Concluiu-se que os resultados deste estudo preliminar de curto prazo sugerem que os pacientes que tomam bifosfonatos por via oral podem esperar um resultado satisfatório com evidências de reparo perirradicular após tratamento endodôntico.

Foram descritos dois casos clínicos de osteonecrose associada a bifosfonatos nos quais o tratamento endodôntico cirúrgico ou não-cirúrgico estavam associados com o localmente. Nos dois casos, o tratamento endodôntico foi realizado por se suspeitar de possível causa odontogênica da dor, não sendo diretamente relacionados à causa do desenvolvimento da osteonecrose dos maxilares (SARATHY et al., 2005).

\section{Discussão}

Os bifosfonatos, a partir do seu efeito sobre os osteoclastos, direta ou indiretamente, conseguem inibir a reabsorção óssea, por meio da redução da remodelação. Este efeito faz com que a densidade mineral óssea dos pacientes seja aumentada, reduzindo assim o índice de fraturas ósseas $e$ melhorando a qualidade de vida (RODAN; FLEISCH, 1996; RODAN, 1998; RESZKA; RODAN, 2003; FERREIRA-JUNIOR et al., 2007; CARTSOS et al., 2008; KWAK et al., 2009; WEINSTEIN et al., 2009)

A diminuição da remodelação leva à renovação de osso em períodos mais longos. Como consequência tem-se ossos mais mineralizados e envelhecidos, podendo levar a micro danos e como consequência a ocorrência de ocorrência de osteonecrose (RODAN; FLEISCH, 1996; RODAN, 1998; GUYTON; HALL, 2006; KWAK et al., 2009; WEINSTEIN et al., 2009).

Os estimuladores de reabsorção óssea testados foram inibidos pelos bifosfonatos. Tal efeito pode agir inibindo a defesa orgânica, já que estão também envolvidos no processo inflamatório necessário ao mecanismo de defesa do organismo que desencadeia a formação das periodontites apicais. Neste caso, o processo de defesa do organismo poderia ser comprometido pelo processo infeccioso não se manter restrito à região dental e periapical, mas se espalhar pelo osso (SHINODA et al., 2008; KWAK et al., 2009; TOUSSAINT et al., 2009).

Estudos em roedores encontraram diminuição na formação óssea no reparo de feridas cirúrgicas no período inicial de 5 a 10 dias pelo uso de bifosfonatos (HIKITA et al., 2009; AGUIRRE et al., 2010; KOBAYASHI et al., 2010), e diminuição da angiogênese (AGUIRRE et al., 2010; KOBAYASHI et al., 2010). Já em períodos mais tardios do reparo, compreendendo de 14 a 70 dias, a formação óssea e angiogênese apresentavam-se níveis normais (HIKITA et al., 2009; AGUIRRE et al., 2010; XIONG et al., 2010). Em galinhas, o uso de bifosfonatos teve efeito positivo sobre a formação óssea no período de 7 dias (WEI et al., 2011). Em cães, a quantidade de osso formado após 4 e 8 semanas se manteve em nível normal. O que diferenciou o reparo para o grupo tratado com bifosfonatos foi o tipo de osso formado, tendo neste grupo o osso trabecular permanecido no alvéolo e sem formação de barreira cortical na região apical (ALLEN et al., 2001).

Os estudos em animais mostraram possíveis efeitos dos bifosfonatos no processo de reparo de feridas ósseas (ALLEN et al., 2001; HIKITA et al., 2009; AGUIRRE et al., 2010; KOBAYASHI et al., 2010). Entretanto, a falta de um modelo de estudo em animais dificulta a extrapolação destes resultados para a realidade clínica (ALLEN, 2011). Mais estudos se fazem necessários na determinação de um modelo animal para estudo dos efeitos dos bifosfonatos, assim como para a determinação destes efeitos e se apresentam implicações à prática clínica.

Já a perda óssea foi reduzida pelo uso de bifosfonatos em processos que induzem a reabsorção óssea, como movimentação ortodôntica e periodontites apicais (XIONG et al., 2007; SHINODA et al., 2008; XIONG et al., 2010). Há de se enfatizar que uma menor perda óssea, no caso das periodontites, não necessariamente significa melhor ou pior resultado em relação ao reparo de lesões. A reabsorção óssea faz parte do processo de formação da lesão inflamatória periapical, sendo esta necessária à defesa do organismo frente à infecção endodôntica (ESTRELA, 2009). Fazem-se necessários estudos que busquem identificar a influência da redução de perda óssea no reparo de periodontites apicais.

Em humanos, estudo com bifosfonatos por via oral, normalmente utilizada no tratamento de osteoporose, não observou diferença estatística entre o reparo de periodontites apicais em grupos de pacientes que tomavam bifosfonatos em comparação aos que não faziam uso desta medicação (HSIAO et al., 2009). Contudo, deve-se enfatizar que o tamanho da amostra, o curto período de acompanhamento, o uso de protocolos não padronizados e a utilização de radiografias bidimensionais para avaliar o reparo apical são vieses que podem ter influenciado no resultado final do trabalho. Mais estudos se mostram necessários para avaliar o efeito destes medicamentos no prognóstico do tratamento endodôntico, e em especial no processo de reparo de periodontites apicais, em humanos (XIONG et al., 2007; HSIAO et al., 2009; RIPAMNONTI et al., 2009).

Quanto ao uso de bifosfonatos por via intravenosa, há muitas variáveis que podem interferir no tratamento endodôntico. Normalmente esta via de administração é utilizada para o tratamento em oncologia, com doses bem mais elevadas. A condição imunológica deficitária do paciente, os tratamentos concomitantes a que este é submetido e os próprios efeitos do câncer podem estar associados e dificultar ou mesmo impossibilitar a defesa do organismo e o reparo de lesões periapicais. Deste modo, pacientes que serão submetidos a tratamento oncológico quimioterápico devem inicialmente passar por avaliação odontológica a fim de se realizar o tratamento necessário da forma mais rápida e eficiente possível e evitar a necessidade do tratamento odontológico durante ou logo após o tratamento quimio e/ou radioterápico (KATZ, 2005; SARATHY et al., 2005; FERREIRA JUNIOR et al., 2007).

O principal efeito adverso dos bifosfonatos é, sem dúvida, a osteonecrose dos maxilares. Esta acomete mais a pacientes que fazem uso destes medicamentos por via endovenosa, diretamente relacionada à dose e potencia dos medicamentos. Também tem sido relatada em pacientes que fizeram uso de bifosfonatos por via oral, mas em frequência consideravelmente inferior. A presença desta patologia contraindica a realização de procedimentos cirúrgicos e demais procedimentos que possam disseminar microrganismos. Trata-se de uma patologia de difícil tratamento e cuja ocorrência deve ser evitada ao máximo com a realização de medidas preventivas antes da administração de bifosfonatos, como a previa realização de tratamento odontológico e eliminação de focos de contaminação. $\mathrm{Na}$ necessidade de intervenção, o tratamento endodôntico é preferível a cirurgias, pois há maior capacidade de manter a infecção restrita ao local, ou seja, o sistema de canais radiculares (MARX et al., 2005; FERREIRA JUNIOR et al., 2007; LEVIN et al., 2007; EDWARDS et al., 2008; ESTILO et al., 2008). Fica clara, portanto, a importância de manutenção do comprimento de trabalho dentro do canal radicular, ou seja, antes do limite cemento-dentina-canal, evitando assim a disseminação de microrganismos (ESTRELA, 2009) e evitando a contaminação do osso em pacientes que façam ou fizeram uso de bifosfonatos de modo crônico ou em altas doses.

\section{Considerações Finais}

Os mecanismos de ação dos bifosfonatos incluem redução da remodelação óssea e inibição de mediadores da reabsorção, os mesmos mediadores do processo de inflamação. 
Os bifosfonatos demonstram, analisando-se seus mecanismos de ação, potencial para influenciar no reparo de periodontites. Estudos devem ser realizados a fim de estabelecer uma relação causa-efeito entre o uso de bifosfonatos e o processo de reparo de lesões ósseas.

\section{Referências}

ABUGHAZALEH, K.; KAWAR, N. Osteonecrosis of the jaws: what the physician needs to know: practical considerations. Dis. Mon., Chicago, v. 57, no. 4, p. 231-241, Apr. 2011.

AGHALOO, T.L.; FELSENFELD, A.L.; TETRADIS, S. Osteonecrosis of the jaw in a patient on denosumab. J. Oral Maxillofac. Surg., Philadelphia, v. 68, no. 5, p. 959-963, May 2010.

AGUIRRE, J.I. et al. Effects of alendronate on bone healing after tooth extraction in rats. Oral Dis., Houndmills, v. 16 , no. 7 , p. $674-$ 685, Oct. 2010.

ALLEN, M.R. The effects of bisphosphonates on jaw bone remodeling, tissue properties, and extraction healing. Odontology, Tokyo, v. 99, no. 1, p. 8-17, Jan. 2011.

ALLEN, M.R. et al. Morphological assessment of basic multicellular unit resorption parameters in dogs shows additional mechanisms of bisphosphonate effects on bone. Calcif. Tissue Int., Berlin, v. 86, no. 1, p. 67-71, Jan. 2010.

ALLEN, M.R. et al. Compromised osseous healing of dental extraction sites in zoledronic acid-treated dogs. Osteoporos. Int., London, v. 22, no. 2, p. 693-702, Feb. 2001.

ANGELI, A. et al. High prevalence of asymptomatic vertebral fractures in post-menopausal women receiving chronic glucocorticoid therapy: a cross-sectional outpatient study. Bone, New York, v. 39, no. 2, p. 253-259, Aug. 2006.

BOONEKAMP, P.M. et al. Two modes of action of bisphosphonates on osteoclastic resorption of mineralized matrix. Bone Miner., Amsterdam, v. 1, no. 1, p. 27-39, 1986.

BRANDÃO, C.M.R. et al. Treatment of postmenopausal osteoporosis in women: a systematic review. Cad. Saúde Pública, Rio de Janeiro, v. 24, Supl. 4, p. S592-S606, 2008.

BRASIL. Ministério da Saúde. SUS gasta quase R\$ 81 milhões com fraturas em idosos em 2009. Saiba mais! Portal da Saúde. $2010 . \quad$ Disponível em: http://portal.saude.gov.br/portal/saude/visualizar_texto.cfm?idtxt=33 674\&janela=1 Acesso em: 30 abr. 2010.

BURT, M.E.; BRENNAN, M.F. Incidence of hypercalcaemia and malignant neoplasm. Arch. Surg., Chicago, v. 115, no. 6, p. 704707, June 1980.

CANALIS, E. et al. Glucocorticoid-induced osteoporosis: pathophysiology and therapy. Osteoporos. Int., London, v. 18, no. 10, p. 1319-1328, Oct. 2007.

CARTSOS, V.M.; ZHU, S.; ZAVRAS, A.I. Bisphosphonate use and the risk of adverse jaw outcomes. A medical claims study of 714,217 people. J. Am. Dent. Assoc., Chicago, v. 139, no. 1, p. 23-30, Jan. 2008.

CARVALHO, A. Osteonecrose da mandíbula associada a bifosfonatos intravenosos em doentes oncológicos. Acta. Med. Port., Lisboa, v. 21, no. 5, p. 505-510, set./out. 2008.
CHEN, Y.C.; SOSNOSKI, D.M.; MASTRO, A.M. Breast cancer metastasis to the bone: mechanisms of bone loss. Breast Cancer Res., London, v. 12, no. 6, p. 215-226, Dec. 2006.

COLELLA, G.; CAMPISI, G.; FUSCO, V. American Association of Oral and Maxillofacial Surgeons: position paper on bisphosphonaterelated osteonecrosis of the jaws-2009 update. J. Oral Maxillofac. Surg., Philadelphia, v. 67 , no. 12, p. 2698-2699, Dec. 2009.

CUNDY, T.; BOLLAND, M. Paget disease of bone. Trends Endocrinol. Metab., New York, v. 19, no. 7, p. 246-253, Sept. 2008.

DORE, F. et al. Bone scintigraphy and SPECT/CT of bisphosphonate-induced osteonecrosis of the jaw. J. Nucl. Med., New York, v. 50, no. 1, p. 30-35, Jan. 2009.

EDWARDS, B.J. et al. Updated recommendations for managing the care of patients receiving oral bisphosphonate therapy. An advisory statement from the American Dental Association Council on Scientific Affairs. J. Am. Dent. Assoc., Chicago, v. 139, no. 12, p. 1674-1677, Dec. 2008.

ESTILO, C.L. et al. Osteonecrosis of the maxilla and mandible in patients with advanced cancer treated with bisphosphonate therapy. Oncologist, Dayton, v. 13, no. 8, p. 911-920, Aug. 2008.

ESTRELA, C. Endodontic science. São Paulo-SP: Artes Médicas, 2009. 1104p.

FERREIRA JUNIOR, C.D.; CASADO, P.L.; BARBOZA, L.S.P. Osteonecrose Associada aos Bifosfonatos na Odontologia. R. Periodontia, Belo Horizonte-MG, v. 17, no. 4, p. 24-30, dez. 2007.

FREIBERGER, J.J. Utility of hyperbaric oxygen in treatment of bisphosphonate-related osteonecrosis of the jaws. J. Oral Maxillofac. Surg., Philadelphia, v. 67, no. 5 Suppl., p. 96-106, May 2009.

GROSSMANN, M. et al. Bone metabolic health in patients with nonmetastatic prostate cancer who are receiving androgen deprivation therapy. Med. J. Aust., Sydney, v. 194, no. 6, p. 301-306, Mar. 2011.

GUISE, T.A.; BRUFSKY, A.; COLEMAN, R.E. Understanding and optimizing bone health in breast cancer. Curr. Med. Res. Opin., London, v. 26, Suppl. 3, p. 3-20, Dec. 2010.

GUYTON, C.A.; HALL. J.E. Tratado de fisiologia médica. 11th ed. Rio de Janeiro-RJ: Guanabara Koogan, 2006. 1264p.

HIKITA, $H$. et al. Bisphosphonate administration prior to tooth extraction delays initial healing of the extraction socket in rats. J. Bone Miner. Metab., Tokyo, v. 27, no. 6, p. 663-672, June 2009.

HSIAO, A.; GLICKMAN, G.; HE, J. A Retrospective clinical and radiographic study on healing of periradicular lesions in patients taking oral bisphosphonates. J. Endod., Chicago, v. 35, no. 11, p. 1525-1528, Nov. 2009.

HUGHES, D.E. et. al. Inhibition of osteoclast-like cell formation by bisphosphonates in long-term cultures of human bone marrow. $\mathbf{J}$. Clin. Invest., New York, v. 83, no. 6, p. 1930-1935, June 1989.

KATZ, H. Endodontic implications of bisphosphonate-associated osteonecrosis of the jaws: a report of three cases. J. Endod., Chicago, v. 31, no. 11, p. 831-834, Nov. 2005. 
KHOSLA, S. et al. Bisphosphonate-associated osteonecrosis of the jaw: report of a task force of the American Society for Bone and Mineral Research. J. Bone Miner. Res. New York, v. 22, no. 10, p. 1479-1491, Oct. 2007.

KOBAYASHI Y. et al. Zoledronic acid delays wound healing of the tooth extraction socket, inhibits oral epithelial cell migration, and promotes proliferation and adhesion to hydroxyapatite of oral bacteria, without causing osteonecrosis of the jaw, in mice. J. Bone Miner. Metab., Tokyo, v. 28, no. 2, p. 165-175, Mar. 2010.

KOMATSU, K. et al. Long-term effects of local pretreatment with alendronate on healing of replanted rat teeth. J. Periodont. Res., Copenhagen, v. 43, no. 2, p. 194-200, Apr. 2008.

KWAK, H.B. et al. Risedronate directly inhibits osteoclast differentiation and inflammatory bone loss. Biol. Pharm. Bull., Tokyo, v. 32, no. 7, p. 1193-1198, July 2009.

LEVIN, L.; LAVIV, A.; SCHWARTZ-ARAD, D. Denture-related osteonecrosis of the maxilla associated with oral bisphosphonate treatment. J. Am. Dent. Assoc., Chicago, v. 138, no. 9, p. 12181220, Sept. 2007

LIPTON, A. Implications of bone metastases and the benefits of bone-targeted therapy. Semin. Oncol., New York, v. 37, Suppl. 2 p. S15-S29, Oct. 2010.

MAKRAS, P.; PAPAPOULOS, S.E. Medical treatment of hypercalcaemia. Hormones (Athens), Athens, v. 8, no. 2, p. 83-95 Apr-June 2009.

MARX, R.E. et al. Bisphosphonate-induced exposed bone (osteonecrosis / osteopetrosis) of the jaws: risk factors, recognition, prevention, and treatment. J. Oral. Maxillofac. Surg., New Delhi, v. 63 , no. 11 , p. 1567-1575, Nov. 2005.

MELO, M.D.; OBEID, G. Osteonecrosis of the jaws in patients with a history of receiving bisphosphonate therapy Strategies for prevention and early recognition J. Am. Dent. Assoc., Chicago, v. 136, no. 12, p. 1675-1681, Dec. 2005.

MURAKAMI, $\mathrm{H}$. et al. A possible mechanism of the specific action of bisphosphonates on osteoclasts: tiludronate preferentially affects polarized osteoclasts having ruffled borders. Bone, New York, v. 17, no. 2, p. 137-144, Aug. 1995.

NEVILLE-WEBBE, H.L.; COLEMAN, R.E. Bisphosphonates and RANK ligand inhibitors for the treatment and prevention of metastatic bone disease. Eur. J. Cancer, Oxford, v. 46, no. 7, p. 1211-1222, May 2010

PLASMANS, C.M.T.; KUYPERS, P.H.K.J.W.; SLOOFF, T.J.J Influence of a diphosphonate on the cellular aspect of young bone tissue. Calcif. Tissue Int., Berlin, v. 32, no. 3, p. 247-266, 1980.

RENTSCH, C.A.; CECCHINI, M.G.; THALMANN, G.N. LosS of inhibition over master pathways of bone mass regulation results in osteosclerotic bone metastases in prostate cancer. Swiss Med. Wkly., Basel, v. 139, no. 15-16, p. 220-225, Apr. 2009.

RESZKA, A.A.; RODAN, G.A. Mechanism of action of bisphosphonates. Curr. Osteoporos. Rep., Chicago, v. 1, no. 2, p. 45-52, Sept. 2003.

RIPAMONTI C.I. et al. Decreased occurrence of osteonecrosis of the jaw after implementation of dental preventive measures in solid tumor patients with bone metastases treated with bisphosphonates. The experience of the National Cancer Institute of Milan. Ann. Oncol., Dordrecht, v. 20, no. 1, p. 137-145, Jan. 2009.
RIZZOLI, R. et al. Osteonecrosis of the jaw and bisphosphonate treatment for osteoporosis. Bone, New York, v. 42, no. 5, p. 841 847 , May 2008

RODAN, A.; FLEISCH, H.A. Bisphosphonates: mechanisms of action. J. Clin. Invest., New York, v. 97, no. 12, p.2692-2696, June 1996.

RODAN, G.A. Mechanisms of action of bisphosphonates. Annu. Rev. Pharmacol. Toxicol., San Diego, v. 38, p. 375-388, 1998.

RUSSELL, R.G. et al. Mechanisms of action of bisphosphonates: similarities and differences and their potential influence on clinical efficacy. Osteoporos. Int., London, v. 19, no. 6, p. 733-759, June 2008

SARATHY, A.P.; BOURGEOIS, S.L.; GOODELL, G.G Bisphosphonate-associated osteonecrosis of the jaws and endodontic treatment: two case reports. J. Endod., Chicago, v. 31 no. 10 , p. $759-763$, Oct. 2005.

SARGENT, J.T.; SMITH, O.P. Haematological emergencies managing hypercalcaemia in adults and children with haematological disorders. Br. J. Haematol., Oxford, v. 149, no. 4, p. $465-477$, May 2010

SATO, M., et al. Bisphosphonate action. Alendronate localization in rat bone and effects on osteoclast ultrastructure. J. Clin. Invest. New York, v. 88, no. 6, p. 2095-2105, Dec. 1991

SATO, M.; GRASSER, W. Effects of bisphosphonates on isolated rat osteoclasts as examined by reflected light microscopy. J. Bone Miner. Res., New York, v. 5, no. 1, p. 31-40, Jan. 1990.

SCHENK, R. et al. Effect of ethane-1-hydroxy-1,1-diphosphonate (EHDP) and dichloromethylene diphosphonate (Cl 2 MDP) on the calcification and resorption of cartilage and bone in the tibial epiphysis and metaphysis of rats. Calcif. Tissue Res., Berlin, v. 11, no. 3e, p. 196-214, Mar. 1973.

SCHMIDT, A. et al. Protein-tyrosine phosphatase activity regulates osteoclast formation and function: inhibition by alendronate. Proc. Natl. Acad. Sci. U. S. A., Washington, v. 93, no. 7, p. 3068-3073, Apr. 1996.

SHINODA, $\mathrm{H}$. et al. Pharmacological topics of bone metabolism: a novel bisphosphonate for the treatment of periodontitis. $J$. Pharmacol. Sci., Tokyo, v. 106, no. 4, p. 555-558, Apr. 2008.

SILVERMAN, S.L. Paget disease of bone: therapeutic options. J. Clin. Rheumatol., Philadelphia, v. 14, no. 5, p. 299-305, Oct. 2008.

TERPOS, E.; DIMOPOULOS, M.A.; BERENSON, J. Established role of bisphosphonate therapy for prevention of skeletal complications from myeloma bone disease. Crit. Rev. Oncol. Haematol., Boca Raton, v. 77, Suppl. 1, p. S13-S23, Feb. 2011.

THONG, Y.L. Intracanal bisphosphonate does not inhibit replacement resorption associated with delayed replantation of monkey incisors. Dent. Traumatol., Copenhagen, v. 25, no. 4, p. 386-393, Aug. 2009

TOUSSAINT, N.D.; ELDER, G.J.; KERR, P.G. Bisphosphonates in chronic kidney disease; balancing potential benefits and adverse effects on bone and soft tissue. Clin. J. Am. Soc. Nephrol., Gainesville, v. 4, no. 1, p. 221-233, Jan. 2009.

VASSILOPOULOU-SELLIN, R. et al. Incidence of hypercalcaemia in patients with malignancy referred to a comprehensive cancer 

1993.

WEI, L.L. et al. The influence of a bisphosphonate on bone generation determined using a chick-femur model. Int. Endod. J., Oxford, v. 44, no. 6, p. 550-559, June 2011.

WEINSTEIN, R.S. Clinical practice. Glucocosticoid-induced bone disease. N. Engl. J. Med., Boston, v. 365, no. 1, p. 62-70, July 2011.

WEINSTEIN, R.S. Glucocorticoid-induced osteoporosis. In: Rosen, C. The ASBMR primer on the metabolic bone diseases and disorders of mineral metabolism. 7th ed. Washington: ASBMR, 2008. p. 267-272.

WEINSTEIN, R.S.; ROBERSON, P.K.; MANOLAGAS, S.C. Giant Osteoclast Formation and Long-Term Oral Bisphosphonate Therapy. N. Engl. J. Med., Boston, v. 360, no. 1, p. 53-62, Jan. 2009.

XIONG, H.; PENG, B.; WEI, L.; ZHANG, X.; WANG, L. Effect of an Estrogen-deficient State and Alendronate Therapy on Bone Loss Resulting from Experimental Periapical Lesions in Rats. J. Endod., Chicago, v. 33, no. 11, p. 1304-1308, Nov 2007.

XIONG, $\mathrm{H}$. et al. Effect of alendronate on alveolar bone resorption and angiogenesis in rats with experimental periapical lesions. Int. Endod. J., Oxford, v. 43, no. 6, p. 485-491, June 2010. 